\title{
Integrable minisuperspace models with Liouville field: energy density self-adjointness and semiclassical wave packets
}

\author{
Alexander A. Andrianov ${ }^{1,4, a}$, Chen Lan ${ }^{2, b}$, Oleg O. Novikov ${ }^{1, \mathrm{c}}$, Yi-Fan Wang ${ }^{3, \mathrm{~d}}$ \\ ${ }^{1}$ Saint Petersburg State University, 7/9 Universitetskaya nab., St. Petersburg 199034, Russia \\ ${ }^{2}$ ELI-ALPS Research Institute, Budapesti út 5, 67228 Szeged, Hungary \\ ${ }^{3}$ Institut für Theoretische Physik, Universität zu Köln, Zülpicher Straße 77, 50937 Köln, Germany \\ ${ }^{4}$ Institut de Ciències del Cosmos, Universitat de Barcelona, Martí i Franquès 1, 08028 Barcelona, Spain
}

Received: 24 May 2018 / Accepted: 16 September 2018 / Published online: 27 September 2018

(C) The Author(s) 2018

\begin{abstract}
The homogeneous cosmological models with a Liouville scalar field are investigated in classical and quantum contexts of Wheeler-DeWitt geometrodynamics. In the quantum case of quintessence field with potential unbounded from below and phantom field, the energy density operators are not essentially self-adjoint, and self-adjoint extensions contain ambiguities. Therefore the same classical actions correspond to a family of distinct quantum models. For the phantom field the energy spectrum happens to be discrete. The probability conservation and appropriate classical limit can be achieved with a certain restriction of the functional class. The appropriately localized wave packets are studied numerically using the Schrödinger's norm and a conserved Mostafazadeh's norm introduced from techniques of pseudoHermitian quantum mechanics. These norms give a similar packet evolution that is confronted with analytical classical solutions.
\end{abstract}

\section{Introduction}

Cosmological models with scalar fields have drawn a lot of attention in the last decades because of investigations on cosmological inflation [1] and dark energy [2], but few of them can be exactly integrated. A universe driven by scalar fields with an exponential potential is dubbed Liouville cosmology, which is one of the well-studied integrable models in cosmology. The power-law expansion of particular solutions and its applications are investigated in e.g. [3-5]. The general classical solutions have been discussed in detail under various

\footnotetext{
a e-mail: a.andrianov@ spbu.ru

be-mail: stlanchen@yandex.ru

c e-mail: o.novikov@spbu.ru

de-mail: yfwang@thp.uni-koeln.de
}

gauge conditions in e.g. [6,7]. The correspondence between Jordan and Einstein frame is studied in [8-13], wherein the Liouville field in the Einstein frame is related to the powerlaw potential in Jordan frame through a conformal transformation combining with a parameter transformation of scalar field. The exactly solvable models with several Liouville scalar fields were developed in $[14,15]$. The appearance of the Lioville cosmologies from higher-dimensional theories, in particular superstring theories and M-theory was studied in $[16,17]$.

General relativity is a theory with constraints, the corresponding Hamiltonian is zero [18-21]. The reason for the vanishing Hamiltonian is the presence of a non-dynamical symmetry, namely diffeomorphism invariance; in other words, the gravitational theory contains redundant degrees of freedom. In the minisuperspace approximation, the redundancy appears in the form of the lapse function $N(t)$. Therefore, to solve the dynamics of the model, it is necessary to introduce a specific gauge condition to eliminate $N(t)[6,14]$. Traditionally, the lapse function is set to unity, such that the universe evolves in cosmic time [22]. However one could eliminate $N(t)$ and avoid an explicit time parametrization to obtain exact solutions of Einstein's equation. This fits well the Wheeler-DeWitt quantum cosmology which does not involve time.

The cosmological models driven by a scalar field with a constant potential may serve as examples of the latter approach $[5,21]$. In these models, the scalar field is a cyclic coordinate, hence the conjugate momentum is integral of motion, and the conservation law can be applied to eliminate the lapse function $N(t)$, such that the modified Friedman equation contains only minisuperspace variables. Inspired by this, we introduce a similar integral of motion in Liouville cosmology of homogeneous and isotropic models [23], in order to eliminate the redundant degrees of freedom. 
With the help of this integral of motion, the classical Friedman equation reduces to a time-independent nonlinear equation, the solution of which can be derived explicitly and describes the trajectory in minisuperspace. This method can also be directly extended to higher dimensional $[24,25]$ and anisotropic models, such as Bianchi-I cosmology considered in [12].

The physical meaning of the formal Wheeler-DeWitt equation and its correspondence with the classical theory can be derived in three steps. The first one is the selection of the space of physical wave functions, usually by endowing proper boundary conditions. In traditional quantum mechanics, crucial properties of the theory depend on the boundary conditions for wave functions, such as the Hermiticity of observables [26], the orthogonality of wave functions (e.g. $[26,27])$ and the conservation of probability, to name a few. A similar situation holds in quantum cosmology [21,28], in which proper boundary conditions have to be specified, such that the solutions of the Wheeler-DeWitt equation, which are not square-integrable, are eliminated from the space of physical wave functions. In this paper we address an important issue encountered at this step. The Hamiltonian operator naively constructed by the canonical quantization in some cosmological models, which are interesting from the phenomenological point of view, including phantom field, happens to be not essentially self-adjoint and its self-adjoint extension is not unique [29-31]. Namely while the clasical action fixes up to the usual ordering ambiguities how the Hamiltonian acts on the localized wavefunctions the evolution over finite amounts of time depends on its behaviour at infinity where extra ambiguity arises. Hence one classical action correspond to a family of distinct quantum models with different quantum evolutions. The cosmological models with similar self-adjointness issues were considered in $[32,33]$.

The second step is to define an inner product on the physical space that would give the conserved probability distribution in quantum cosmology. Since the Wheeler-DeWitt equation is of Klein-Gordon type, the 'probability density' defined by the so-called Klein-Gordon norm is not guaranteed to be positive. While one may restrict consideration to the WKB wavepackets the question arises how to interpret the wavefunction of the universe beyond the WKB region. A resolution of this problem may be provided within the pseudo-Hermitian theory by introducing the Mostafazadeh's norm [34-36]. While we do not treat this norm as the only possible way to tackle the probability problem it may be considered as an useful tool to study the quantum cosmology as a fully consistent quantum theory within restrictions of the minisuperspace approximation.

Finally one has to attribute a proper energy distribution to construct a wave packet [37-39]. For a given initial coordinate distribution of wave packet in minisuperspace, the energy distribution can be calculated, which however is not easy to realize in practice. A common compromise is to choose a Gaussian energy distribution. Then in correspondence with classical theory the probability distribution of the established wave packet should 'centre' at the classical path and follow it as closely as possible apart from turning points.

This paper is organized as follows. In Sect. 2 we briefly elucidate the problem of the quantum particle in the unstable potential $V=-\mathrm{e}^{2 x}$ and the ambiguity of self-adjoint extension of the Hamiltonian operator. In Sect. 3 an integral of motion is introduced for three types of Liouville cosmological models and explicit classical solutions are given in terms of minisuperspace variables. Section 4 introduces the corresponding canonical quantum cosmology and there the physical state space is constructed. As a verification of the results, in Sect. 5 the limit of potential parameter $\lambda$ tending to zero is considered. Section 6 is devoted to the classical-quantum correspondence, in which the wave packets are implemented and the probability distributions are plotted for two kinds of norms. The conclusions Sect. 7 contain some comments on further extensions and applications of the approach adopted in this paper.

\section{Quantum mechanics of a particle in a negative Liouville potential}

To explain the issues that will arise in the quantum cosmological models of interest let us consider the one-dimensional motion of a non-relativistic particle in a Liouville potential which is unbounded from below, described by the Hamiltonian

$H=p^{2}-\mathrm{e}^{2 x}$

This is the special case of the unstable Morse potential considered in detail in [30, Ch. 8.5].

The corresponding time-independent Schrödinger equation is

$\hat{H} \psi:=\left(-\partial_{x}^{2}-\mathrm{e}^{2 x}\right) \psi=E \psi$

For the positive energies $E>0$ the solutions are,

$\psi_{k}=c_{1} \mathrm{~F}_{\mathrm{i} k}\left(\mathrm{e}^{x}\right)+c_{2} \mathrm{G}_{\mathrm{i} k}\left(\mathrm{e}^{x}\right), \quad E=k^{2}$

where,

$$
\begin{aligned}
& \mathrm{F}_{v}(z)=\frac{1}{2} \sec \left(\frac{v \pi}{2}\right)\left[\mathrm{J}_{v}(z)+\mathrm{J}_{-v}(z)\right], \\
& \mathrm{G}_{v}(z)=\frac{1}{2} \csc \left(\frac{v \pi}{2}\right)\left[\mathrm{J}_{v}(z)-\mathrm{J}_{-v}(z)\right]
\end{aligned}
$$


where $J_{v}$ is a Bessel function,

$\mathrm{J}_{v}(z)=\sum_{n=0}^{+\infty} \frac{(-1)^{n}}{n ! \Gamma(n+v+1)}\left(\frac{z}{2}\right)^{2 n+v}$

and functions,

$\mathrm{F}_{v}(z)=\mathrm{F}_{-v}(z), \quad \mathrm{G}_{v}(z)=\mathrm{G}_{-v}(z)$

are defined according to [40]. They have undamped oscillatory behavior as $x \rightarrow-\infty$,

$\mathrm{F}_{\mathrm{i} k}\left(\mathrm{e}^{x}\right) \simeq \sqrt{\frac{2 \tanh \frac{\pi k}{2}}{\pi k}} \cos \left(k x-\delta_{k}\right)+O\left(\mathrm{e}^{2 x}\right)$,

$\mathrm{G}_{\mathrm{i} k}\left(\mathrm{e}^{x}\right) \simeq \sqrt{\frac{2 \operatorname{coth} \frac{\pi k}{2}}{\pi k}} \sin \left(k x-\delta_{k}\right)+O\left(\mathrm{e}^{2 x}\right)$,

and oscillations as $x \rightarrow+\infty$ exponentially decreasing amplitude but accelerating frequency,

$$
\begin{aligned}
& \mathrm{F}_{\mathrm{i} k}\left(\mathrm{e}^{x}\right) \simeq \sqrt{\frac{2}{\pi}} \mathrm{e}^{-x / 2}\left\{\cos \left(\mathrm{e}^{x}-\frac{\pi}{4}\right)+O\left(\mathrm{e}^{-x}\right)\right\}, \\
& \mathrm{G}_{\mathrm{i} k}\left(\mathrm{e}^{x}\right) \simeq \sqrt{\frac{2}{\pi}} \mathrm{e}^{-x / 2}\left\{\sin \left(\mathrm{e}^{x}-\frac{\pi}{4}\right)+O\left(\mathrm{e}^{-x}\right)\right\}
\end{aligned}
$$

Thanks to this behavior both functions should naively contribute to the continuous spectrum. Using the method from [41] one can obtain the following orthogonality relations,

$$
\begin{aligned}
& \int_{-\infty}^{+\infty} \mathrm{d} x \mathrm{~F}_{\mathrm{i} k}\left(\mathrm{e}^{x}\right) \mathrm{F}_{\mathrm{i} l}\left(\mathrm{e}^{x}\right)=\frac{1}{k} \tanh \left(\frac{\pi k}{2}\right) \delta(k-l), \\
& \int_{-\infty}^{+\infty} \mathrm{d} x \mathrm{G}_{\mathrm{i} k}\left(\mathrm{e}^{x}\right) \mathrm{G}_{\mathrm{i} l}\left(\mathrm{e}^{x}\right)=\frac{1}{k} \operatorname{coth}\left(\frac{\pi k}{2}\right) \delta(k-l) .
\end{aligned}
$$

However both of these functions $\mathrm{F}_{\mathrm{i} k}\left(\mathrm{e}^{x}\right)$ and $\mathrm{G}_{\mathrm{i} l}\left(\mathrm{e}^{x}\right)$ can not be included into the continuous spectrum of a self-adjoint operator simultaneously as they are not orthogonal even when $k \neq l$ is different. Nevertheless, we note that their symmetrized scalar product vanishes,

$\int_{-\infty}^{+\infty} \mathrm{d} x\left[\mathrm{~F}_{\mathrm{i} k}\left(\mathrm{e}^{x}\right) \mathrm{G}_{\mathrm{i} l}\left(\mathrm{e}^{x}\right)+\mathrm{G}_{\mathrm{i} k}\left(\mathrm{e}^{x}\right) \mathrm{F}_{\mathrm{i} l}\left(\mathrm{e}^{x}\right)\right]=0$.

For negative energies $E \leq 0$ one naively obtains the continuous spectrum of square-integrable solutions,

$\tilde{\psi}_{\mu}=\sqrt{2 \mu} \mathrm{J}_{\mu}\left(\mathrm{e}^{x}\right), \quad E=-\mu^{2}$.

Similarly to the part of the spectrum with $E>0$, not all of these wavefunctions can be included into the spectrum of a self-adjoint operator because they are not orthogonal for different values of $\mu$ in general [42],

$\int_{-\infty}^{+\infty} \mathrm{d} x \mathbf{J}_{\mu}\left(\mathrm{e}^{x}\right) \mathrm{J}_{v}\left(\mathrm{e}^{x}\right)=2 \frac{\sin \left[\frac{\pi}{2}(\mu-v)\right]}{\pi\left(\mu^{2}-v^{2}\right)}$

These peculiarities are caused by the operator $\hat{H}$, as defined on the standard domain of $\hat{p}^{2}$, being not essentially self-adjoint. Thus it actually describes a family of different self-adjoint extensions that are indistinguishable on sufficiently localized smooth functions but generate different unitary evolutions. Since this important topic is often neglected in the quantum mechanics courses we elucidate few important facts here and refer to [29-31] for details.

In infinite dimensional Hilbert spaces it is too restrictive to demand that the domain of the operator $\mathscr{D}(\hat{A})$ covered the whole Hilber space $\mathscr{H}$. Therefore operators including observables are usually defined on the domains that are merely dense in $\mathscr{H}$ i.e. any element in the Hilbert space can be obtained as a limit of some sequence of elements in $\mathscr{D}(\hat{A})$. For example the operator $\hat{p}^{2}$ can not be defined on the whole $L^{2}(\mathbb{R})$ but is symmetric on the domain of all 'bumps' infinitely differentiable functions with compact support, $\mathscr{C}_{c}^{\infty}$.

However this leads to the following pitfall. Even if its domain is dense a symmetric operator $\hat{A}$ such that,

$(\psi, \hat{A} \chi)=(\hat{A} \psi, \chi), \quad \forall \psi, \chi \in \mathscr{D}(\hat{A})$,

does not in general possess important properties like spectral theorem and reality of eigenvalues. For $\hat{A}$ to be self-adjoint its adjoint $\hat{A}^{\dagger}$ defined as,

$\left(\psi, \hat{A}^{\dagger} \chi\right)=(\hat{A} \psi, \chi)$,

should have the same domain $\mathscr{D}\left(\hat{A}^{\dagger}\right)=\mathscr{D}(\hat{A})$. However in general the domain of $\hat{A}^{\dagger}$ is larger than the domain of $\hat{A}$. In many cases this happens because $\mathscr{D}(\hat{A})$ is selected to be too small and it is possible to find the self-adjoint operator called self-adjoint extension on a larger domain that equals to $\hat{A}$ on the original domain. If such extension is unique $\hat{A}$ is said to be essentially self-adjoint. But in general the operator $\hat{A}$ has many self-adjoint extenstions. This should not be considered as a pathology, rather the original definition of $\hat{A}$ happens to be incomplete and provides merely a local description of many different self-adjoint operators each generating its own unitary evolution.

For non-singular potentials bounded from below the Hamiltonian is essentially self-adjoint. However this is not a case for Eq. (1). It shows itself in the existence of squareintegrable solutions of Eq. (2) with complex $E$. For example for $E_{ \pm}= \pm 2 \mathrm{i}$ one gets,

$\psi_{ \pm}=C_{ \pm} \mathrm{J}_{1 \pm \mathrm{i}}\left(\mathrm{e}^{x}\right)+\tilde{C}_{ \pm} \mathrm{J}_{-1 \mp \mathrm{i}}\left(\mathrm{e}^{x}\right)$, 
The dimensions of the subspaces of solutions corresponding to complex $E$ with $\operatorname{Im} E>0$ and $\operatorname{Im} E<0$ are known as deficiency indices $n_{+}$and $n_{-}$respectively. If $n_{+}=n_{-}=0$ (i.e. there are no such solutions) the operator is essentially self-adjoint, that is its self-adjoint extension is unique. If $n_{+} \neq n_{-}$no self-adjoint extension exists. In our case the square-integrability requires $\tilde{C}_{ \pm}=0$ however $C_{ \pm} \neq 0$ is allowed. Therefore $n_{+}=n_{-}=1$. According to the Weylvon Neumann theorem [29] this means that a single parameter family of self-adjoint extensions exists.

The functions $\tilde{\psi}_{\mu}$ are square integrable but don't belong to $\mathscr{C}_{c}^{\infty}$. As result the $\hat{p}^{2}$ and $\hat{H}$ are not generally symmetric on these solutions,

$$
\begin{aligned}
& \int_{-\infty}^{+\infty} \mathrm{d} x \tilde{\psi}_{\mu}^{*}(x)\left[\hat{H} \tilde{\psi}_{v}(x)\right]-\int_{-\infty}^{+\infty} \mathrm{d} x\left[\hat{H} \tilde{\psi}_{\mu}(x)\right]^{*} \tilde{\psi}_{v}(x) \\
& =\frac{2}{\pi} \sqrt{\mu \nu} \sin \left[\frac{\pi}{2}(\mu-v)\right] .
\end{aligned}
$$

To extend the domain of $\hat{H}$ conserving its symmetricity we consider the new functional class bigger than $\mathscr{C}_{c}^{\infty}$ with a specific oscillatory behavior as $x \rightarrow+\infty$,

$\psi \sim \mathrm{e}^{-x / 2} \cos \left(\mathrm{e}^{x}-\frac{\pi}{2} a-\frac{\pi}{4}\right)$

where $a$ is an arbitrary parameter $a \in[0,2)$. For $E>0$ using Eqs. (10), (11) we then get non-degenerate continuous spectrum,

$$
\begin{aligned}
\Xi_{k}^{(a)}(x)= & \mathscr{N}_{k}^{(a)}\left[\mathrm{F}_{\mathrm{i} k}\left(\mathrm{e}^{x}\right) \cos \frac{\pi a}{2}+\mathrm{G}_{\mathrm{i} k}\left(\mathrm{e}^{x}\right) \sin \frac{\pi a}{2}\right] . \\
\left(\mathscr{N}_{k}^{(a)}\right)^{-2}= & \frac{1}{k^{2}} \tanh ^{2}\left(\frac{\pi k}{2}\right) \cos ^{2} \frac{\pi a}{2} \\
& +\frac{1}{k^{2}} \operatorname{coth}^{2}\left(\frac{\pi k}{2}\right) \sin ^{2} \frac{\pi a}{2},
\end{aligned}
$$

whereas for $E \leq 0$ using 10.7.2 from [43] we obtain the discrete spectrum,

$\Phi_{n}^{(a)}(x)=\sqrt{2(2 n+a)} \mathrm{J}_{2 n+a}\left(\mathrm{e}^{x}\right), \quad E=-(2 n+a)^{2}$.

The resulting full spectrum forms orthonormal set,

$$
\begin{aligned}
& \int_{-\infty}^{+\infty} \mathrm{d} x\left[\Phi_{n}^{(a)}(x)\right]^{*} \Phi_{m}^{(a)}(x)=\delta_{n m}, \\
& \int_{-\infty}^{+\infty} \mathrm{d} x\left[\Phi_{n}^{(a)}(x)\right]^{*} \Xi_{k}^{(a)}(x)=0, \\
& \int_{-\infty}^{+\infty} \mathrm{d} x\left[\Xi_{k}^{(a)}(x)\right]^{*} \Xi_{l}^{(a)}(x)=\delta(k-l) .
\end{aligned}
$$

It is interesting that the discreteness of the spectrum for $E<0$ and the non-degeneracy of the continuous spectrum for $E>0$ makes the abyss of the potential at large positive $x$ analogous to a reflecting wall. The classical trajectories for the particle described by $H$ reach infinity in finite time. Therefore in the first WKB approximation, the Gaussian wave packet also reaches the infinity in finite time. The subsequent motion of the particle may be described as a bounce from infinity. The non-uniqueness of the self-adjoint extension for $\hat{H}$ may be understood intuitively in the following way. After crossing over infinity the wave function may be multiplied by an arbitrary phase factor $\mathrm{e}^{2 \pi \mathrm{i} a}$ without losing the conservation of probability. Thus we have a family of unitary evolution operators generated by different self-adjoint extensions of $\hat{H}$ that locally are indistinguishable however differ at finite times.

Another way, perhaps more physical, to understand this non-uniqueness is to consider the regularized potential, for example introducing an infinitely high wall at $x=L$ that forms a potential well with the fall of the potential at large $x$ playing the role of another wall,

$\left[-\partial_{x}^{2}-\mathrm{e}^{2 x}\right] \psi=E \psi,\left.\quad \psi\right|_{x=L}=0$

Even in the limit $L \rightarrow+\infty$ the energy levels for $E<0$ stay apart from each other and the spectrum remains to be discrete. The non-uniqueness of the self-adjoint extension takes the form of the regularization-dependence. The parameter $a$ can be shown to be equal to,

$$
\frac{\pi a}{2}=\left(\mathrm{e}^{L}-\frac{3 \pi}{4}\right) \quad \bmod \pi
$$

\section{Classical solutions of Liouville cosmology}

Consider a Friedmann-Lemaître model minimally coupled with a spatially isotropic and homogeneous Liouville field. The Friedmann-Lemaître- Robertson-Walker (FLRW) metric is

$\mathrm{d} s^{2}=N^{2}(t) \mathrm{d} t^{2}-\mathrm{e}^{2 \alpha(t)} \mathrm{d} \mathbf{x}^{2}$,

where $N(t)$ is the lapse function, and $a(t)=\exp \alpha(t)$ the cosmological scale factor; moreover, the scalar field is a function only of time, $\phi=\phi(t)$. With $\varkappa=8 \pi G, \sigma= \pm 1$ and $\lambda \in \mathbb{R}$, the minisuperspace action reads

$S(\lambda)=\int \mathrm{d} t N \mathrm{e}^{3 \alpha}\left(-\frac{3}{\varkappa} \frac{\dot{\alpha}^{2}}{N^{2}}+\sigma \frac{\dot{\phi}^{2}}{2 N^{2}}-V \mathrm{e}^{\lambda \phi}\right)$,

where $\sigma=+1$ gives a quintessence model [44], and $\sigma=-1$ is dubbed as a phantom model [45]. From Eq. (30) one readily derives the Hamiltonian density

$\mathscr{H}(\lambda)=N \mathrm{e}^{-3 \alpha}\left(-\frac{\varkappa}{12} p_{\alpha}^{2}+\sigma \frac{1}{2} p_{\phi}^{2}+V \mathrm{e}^{6 \alpha+\lambda \phi}\right)$, 
in terms of $\alpha$ and $\phi$, as well as their canonical momenta

$p_{\alpha}=-\mathrm{e}^{3 \alpha} \frac{6}{\varkappa} \frac{\dot{\alpha}}{N}, \quad p_{\phi}=\sigma \mathrm{e}^{3 \alpha} \frac{\dot{\phi}}{N} ;$

the significance of stressing $\lambda$ will be elaborated in Sect. (5). It has been shown in $[14,23]$ that

$\omega:=\mathrm{e}^{3 \alpha}\left(\frac{\lambda}{\varkappa} \frac{\dot{\alpha}}{N}+\sigma \frac{\dot{\phi}}{N}\right)$

is an integral of motion, i.e. $\omega$ is a constant on the constraint surface

$\dot{\omega}=-\frac{2}{\lambda} \mathscr{H} \approx 0$,

where $\approx$ represents Dirac's weak equivalence [46-50].

Applying Eq. (33) to the Friedmann equation

$\frac{\dot{\alpha}^{2}}{N^{2}}=\frac{\varkappa}{3}\left(\sigma \frac{\dot{\phi}^{2}}{2 N^{2}}+V \mathrm{e}^{\lambda \phi}\right)$,

one can eliminate the lapse function $N$ and obtain a nonlinear equation

$$
\left(\frac{\mathrm{d} \alpha(\phi)}{\mathrm{d} \phi}\right)^{2}-\sigma \frac{\varkappa}{6}=\frac{3 \omega^{2}}{\varkappa V} \mathrm{e}^{6 \alpha(\phi)+\lambda \phi}\left(\frac{\lambda}{\varkappa} \frac{\mathrm{d} \alpha(\phi)}{\mathrm{d} \phi}+\sigma\right)^{2}
$$

in terms of minisuperspace variables $\alpha$ and $\phi$ only, and $\dot{\alpha} / \dot{\phi}$ has already been replaced by $\mathrm{d} \alpha(\phi) / \mathrm{d} \phi$. Equation (36) can be solved with the help of a change of variables

$x($ or $y):=6 \alpha+\lambda \phi$.

where $x$ is for quintessence and $y$ is for phantom.

Defining

$m_{x}:=-6 \varkappa+\lambda^{2}$,

the solution for a quintessence model $\sigma=+1$ can be divided into two cases:

1. When $m_{x}$ and $V$ are of different sign, one obtains

$$
\mathrm{e}^{6 \alpha+\lambda \phi}=\frac{3 \varkappa \omega^{2}}{-V m_{x}} \operatorname{csch}^{2}\left(\lambda \sqrt{\frac{3}{2 \varkappa}} \alpha+\sqrt{\frac{3 \varkappa}{2}} \phi+c_{1}\right),
$$

where $c_{1}$ is an integration constant associated with the initial conditions. Equation (39) contains two distinct solutions separated by $\lambda \sqrt{\frac{3}{2 \varkappa}} \alpha+\sqrt{\frac{3 \varkappa}{2}} \phi+c_{1}=0$ due to the divergence of $\operatorname{csch} x$ for $x \rightarrow 0$. Both of the solutions can be interpreted as an expansion model, see e.g. Fig. 2.
For $\omega=0$, one recovers the power-law special solution or $\alpha \propto \phi$ in [5].

2. When $m_{x}$ and $V$ are of the same sign, one has

$$
\mathrm{e}^{6 \alpha+\lambda \phi}=\frac{3 \varkappa \omega^{2}}{V m_{x}} \operatorname{sech}^{2}\left(\lambda \sqrt{\frac{3}{2 \varkappa}} \alpha+\sqrt{\frac{3 \varkappa}{2}} \phi+c_{1}\right),
$$

this trajectory contains a single turning point in finite domain of minisuperspace.

As for the second case the quantization is straightforward we will concentrate on the first case.

Similar to Eq. (38), one can define

$m_{y}:=+6 \varkappa+\lambda^{2}>0$

for phantom model with $\sigma=-1$. The solution reads

$\mathrm{e}^{6 \alpha+\lambda \phi}=\frac{3 \varkappa \omega^{2}}{V m_{y}} \sec ^{2}\left(\lambda \sqrt{\frac{3}{2 \varkappa}} \alpha-\sqrt{\frac{3 \varkappa}{2}} \phi+c_{2}\right)$,

where $c_{2}$ is another integration constant. Equation (42) contains a infinite family of distinct solutions separated by two types of cosmological singularities at infinity, due to the periodic divergences of sec function, see Fig. 1. These three results Eqs. (39), (40) and (42) can also be obtained through Hamilton-Jacobi (HJ) approach [51], where the nonlinear

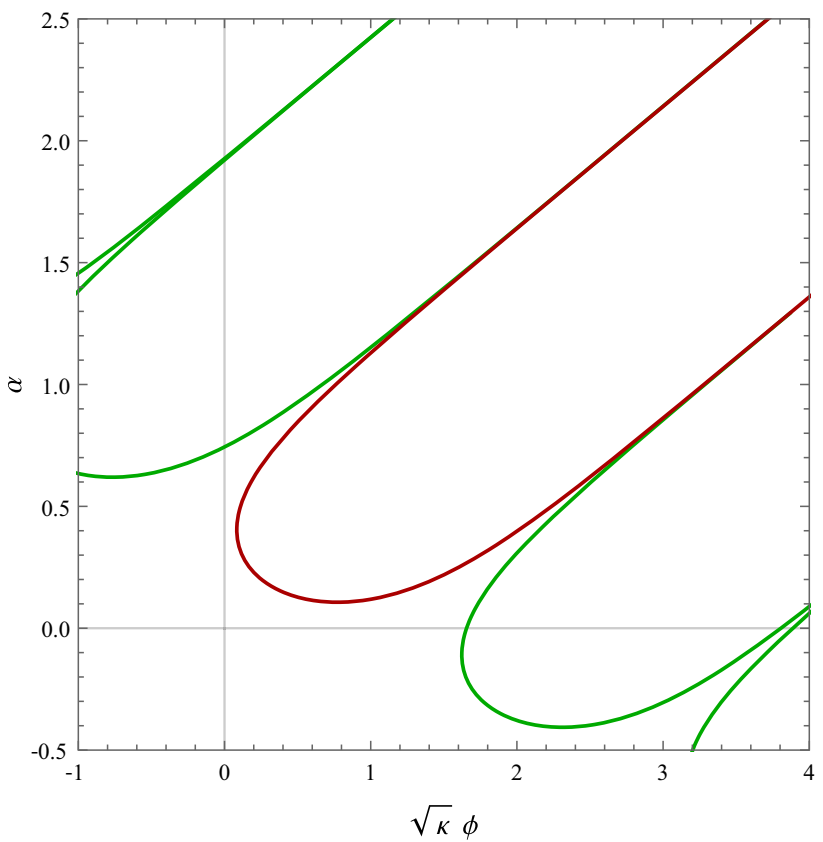

Fig. 1 Multiple solutions contained in Eq. (42) for $\varkappa=1, V=1$, $\lambda=2$ and $A^{2}=160 / 3$. Only one of them is physical, which can be taken to be the red one; the other trajectories (in green) appear due to the ambiguity in the timeless Eq. (42). If a time parameter is chosen, the other branches will disappear 
Eq. (36) is replaced by $\mathrm{HJ}$ equation including $\omega$ as an integral of motion.

With a given set of initial conditions, the universe runs only along one branch. Hence the domain of minisuperspace variables in Eq. (42) has to be restricted

$$
-\frac{\pi}{2}+k \pi \leq \lambda \sqrt{\frac{3}{2 \varkappa}} \alpha-\sqrt{\frac{3 \varkappa}{2}} \phi+c_{2} \leq \frac{\pi}{2}+k \pi, \quad k \in \mathbb{Z},
$$

such that only one trajectory between a pair of singularities is selected. In other words, eliminating time parameter in the classical solution gives rise to Eq. (42) that covers redundant trajectories as well, which should be eliminated by the additional condition Eq. (43). For simplicity, one can choose $c_{1} \equiv 0, k \equiv 0$ and obtain

$$
-\frac{\pi}{2} \leq \lambda \sqrt{\frac{3}{2 \varkappa}} \alpha-\sqrt{\frac{3 \varkappa}{2}} \phi \leq \frac{\pi}{2},
$$

which could be applied as a boundary condition in quantum theory. According to DeWitt's criterion [52] , the wave functions must vanish at classical singularities. This restriction on the classical domain of variables affords the possibility to determine the ambiguity of self-adjoint extension, if one prefers to fix the periodicity of wave function with respect to $\tau$. See Sect. 4.

\section{Dirac quantization of Liouville cosmology}

\subsection{Inner product and probabilities}

On of the basic building blocks of any quantum model is the inner product that allows to assign probabilities. However this is a long standing problem in quantum cosmology due to the Wheeler-DeWitt equation being of the Klein-Gordon type. The naturally conserved Klein-Gordon inner product corresponds to the indefinite norm [20, ch. 5]. Pseudo-Hermitian quantum mechanics [36] provides a cure and will be applied here to reconstruct wave packets based on consistent norms.

Assume that the Wheeler-DeWitt equaton can be written in the form,

$\partial_{\tau}^{2} \psi+\mathbf{D} \psi=0$

The simplest approach is to use the usual Schrödinger inner product,

$\left(\psi_{1}, \psi_{2}\right)_{\mathrm{S}}:=\int_{-\infty}^{+\infty} \mathrm{d} x \psi_{1}^{*}(\tau, x) \psi_{2}(\tau, x)$,

however it is not conserved. On the other hand, the naturally conserved Klein-Gordon inner product,

$$
\begin{aligned}
\left(\psi_{1}, \psi_{2}\right)_{\mathrm{KG}}:= & \int_{-\infty}^{+\infty} \mathrm{d} x\left(\dot{\psi}_{1}^{*}(\tau, x) \psi_{2}(\tau, x)\right. \\
& \left.-\psi_{1}^{*}(\tau, x) \dot{\psi}_{2}(\tau, x)\right),
\end{aligned}
$$

is not suitable to define the probabilities as it is not positivedefinite.

In the pseudo-Hermitian quantum mechanics, an alternative definition of inner product by Mostafazadeh can be adapted from [34,35], where a family of Hilbert spaces with a corresponding pseudo-Hamiltonian were constructed for the Klein-Gordon equation (45). The solution of Eq. (45) are endowed with, again, the Schrödinger $L^{2}(\mathbb{R})$ inner product in Eq. (46), and $\mathbf{D}$ (not necessarily independent of $\tau$ !) is required to be Hermitian with eigenfunctions and nonnegative eigenvalues

$\mathbf{D} \psi_{n}=v_{n}^{2} \psi_{n}$.

The Mostafazadeh inner product of the new Hilbert space, which features time-translational invariance with respect to $\tau$, can be chosen to be

$\left(\psi_{1}, \psi_{2}\right)_{\mathrm{M}}:=\frac{1}{2 \mu}\left[\left(\psi_{1}, \mathbf{D}^{+1 / 2} \psi_{2}\right)_{\mathrm{S}}+\left(\dot{\psi}_{1}, \mathbf{D}^{-1 / 2} \dot{\psi}_{2}\right)_{\mathrm{S}}\right]$,

in which $\mu$ is a normalizing constant, $\dot{\psi}:=\partial_{\tau} \psi$, and $\mathbf{D}^{\gamma}$ is defined by the spectral decomposition

$\mathbf{D}^{\gamma}:=\sum_{n} v_{n}^{2 \gamma} \mathbf{P}_{n}, \quad \mathbf{P}_{n} \psi:=\psi_{n}\left(\psi_{n}, \psi\right)_{\mathrm{S}}$.

Equation (49) is manifestly positive-definite, but its integrand $\varrho$ is, in general, complex. Luckily, a non-negative density

$\rho:=\frac{1}{2 \mu}\left[\left|\mathbf{D}^{+1 / 4} \psi\right|^{2}+\left|\mathbf{D}^{-1 / 4} \dot{\psi}\right|^{2}\right]$

can be defined whose integral gives the Mostafazadeh inner product

$\int \mathrm{d} \mathbf{x} \rho(\mathbf{x}) \equiv(\psi, \psi)_{\mathrm{M}}=: \int \mathrm{d} \mathbf{x} \varrho(\mathbf{x})$.

Therefore $\rho$ is a good candidate for a probability density in the minisuperspace.

\subsection{Quintessence field}

In addition to Eq. (37), a further transformation

$\tau:=\frac{6 \varkappa}{m_{x}}\left(\frac{\lambda}{\varkappa} \alpha+\phi\right)$, 
is to be performed in order to separate the variables, which is related to $\omega$ by

$\omega=\frac{1}{\tilde{N}} \frac{\mathrm{d} \tau}{\mathrm{d} t}, \quad \tilde{N}:=\frac{6 \varkappa}{m_{x}} N \mathrm{e}^{-3 \alpha}$.

Because of Eq. (54), $\tau$ can be treated as the time of a Klein-Gordon-type equation and $\omega$ as its Fourier conjugate. The Hamiltonian in Eq. (31) then becomes

$\mathscr{H}_{x}=N \mathrm{e}^{\frac{3 \varkappa x}{m_{x}}-\frac{\lambda \tau}{2}}\left(-\frac{3 \varkappa}{m_{x}} p_{\tau}^{2}+\frac{m_{x}}{2} p_{x}^{2}+V \mathrm{e}^{x}\right)$,

which is of Klein-Gordon form. Promoting the canonical variables to operators in the position representation

$$
\tau \rightarrow \tau, \quad x \rightarrow x ; \quad p_{\tau} \rightarrow-\mathrm{i} \hbar \frac{\partial}{\partial \tau}, \quad p_{x} \rightarrow-\mathrm{i} \hbar \frac{\partial}{\partial x}
$$

one can obtain the Wheeler-DeWitt equation

$$
\left(\frac{3 \varkappa \hbar^{2}}{m_{x}} \partial_{\tau}^{2}-\frac{m_{x} \hbar^{2}}{2} \partial_{x}^{2}+V \mathrm{e}^{x}\right) \Psi(\tau, x)=0,
$$

which is Eq. (45) with

$\mathbf{D}=-\frac{\hbar^{2} m_{x}^{2}}{6 x} \partial_{x}^{2}+\frac{V m_{x}}{3 x} \mathrm{e}^{x}$

Its solution can be represented by the Fourier integral

$\Psi(\tau, x)=\int_{-\infty}^{+\infty} \mathrm{d} \omega \mathscr{A}(\omega) \mathrm{e}^{-\frac{\mathrm{i}}{\hbar} \tau \omega} \psi(\omega, x)$,

where $\psi(\omega, x)$ satisfies

$$
\left(-\frac{3 \varkappa \omega^{2}}{m_{x}}-\frac{m_{x} \hbar^{2}}{2} \partial_{x}^{2}+V \mathrm{e}^{x}\right) \psi(\omega, x)=0 .
$$

In order to save the Hermiticity of operators and define meaningful probability densities [20,21] one can demand the solution to be square integrable. The expectation value of a physical observable can be defined naively by

$$
\langle\mathscr{O}\rangle=(\Psi, \hat{\mathscr{O}} \Psi)_{\mathrm{S}}:=\int \mathrm{d} x \Psi^{*}(\tau, x) \hat{\mathscr{O}} \Psi(\tau, x)
$$

When $m_{x} V>0$ the operator is essentially self-adjoint and the quantization proceeds in a straightforward fashion. In contrast when $m_{x} V<0$, the Eq. (60) can be regarded as the stationary Schrödinger equation with negative potential unbounded from below and the corresponding operator is not essentially self-adjoint which is the problem that was considered in detail in Sect. 2. The square-integrable functions can be represented as superpositions of eigenfunctions of,

$$
\begin{aligned}
\Psi(\tau, x)= & \int_{-\infty}^{+\infty} \mathrm{d} \omega \mathscr{A}(\omega) \mathrm{e}^{-\frac{\mathrm{i}}{\hbar} \tau \omega} \Xi_{v}^{(a)}\left(\frac{2}{\hbar} \sqrt{\frac{-2 V}{m_{x}}} \mathrm{e}^{x / 2}\right) \\
& +\sum_{( \pm)} \sum_{n=0}^{+\infty} \mathscr{C}_{n}^{( \pm)} \mathrm{e}^{ \pm \frac{1}{\hbar} \tau\left|\omega_{n}\right|} \Phi_{n}^{(a)}\left(\frac{2}{\hbar} \sqrt{\frac{-2 V}{m_{x}}} \mathrm{e}^{x / 2}\right),
\end{aligned}
$$

where $v$ is given by,

$$
v=2 \frac{\sqrt{6 \varkappa}}{\hbar}\left|\frac{\omega}{m_{x}}\right|
$$

and the functions $\Xi_{v}^{(a)}$ and $\Phi_{n}^{(a)}$ are defined in Eqs. (22) and (24) respectively. The solution contains arbitrary parameter $a \in[0,2)$ specifying the self-adjoint extension. The first part of the wave function corresponds to the solution of Eq. (60) with positive $\omega^{2}>0$, while the second is derived from same equation with negative $\omega^{2}<0$. The discrete purely imaginary $\omega_{n}$

$2 n+a=2 \frac{\sqrt{6 x}}{\hbar}\left|\frac{\omega_{n}}{m_{x}}\right|$

are required for completeness and hermiticity, but they produce growing and decreasing modes, which are not compatible with conservation neither of the Klein-Gordon norm Eq. (47) nor of the Mostafazadeh norm Eq. (49). It is worth noting that these modes also violate the classical restriction $\omega^{2}>0$ imposed by reality of metric and field variables in Eq. (40). It will be shown below that the wave packets along the correct classical trajectories can be constructed only from the continuous spectrum $\Xi_{\mathrm{i} v}^{(a)}$. Thus we conclude that on the physical space there's no contribution from the discrete spectrum, i.e.

$\mathscr{C}_{n}^{( \pm)}=0$

as a result in the quantum model both unitary evolution and correct classical limit can be guaranteed.

\subsection{Phantom field}

A transformation similar to the quintessence case

$\tau:=\frac{6 \varkappa}{m_{y}}\left(\frac{\lambda}{\varkappa} \alpha-\phi\right)$ 
can be made for phantom, such that the Hamiltonian in Eq. (31) becomes

$$
\mathscr{H}_{y}=N \mathrm{e}^{-\frac{\lambda \tau}{2}-3 \frac{\varkappa y}{m_{y}}}\left(-\frac{3 \varkappa}{m_{y}} p_{\tau}^{2}-\frac{m_{y}}{2} p_{y}^{2}+V \mathrm{e}^{y}\right) .
$$

The Wheeler-DeWitt equation then reads

$$
\left(\frac{3 \varkappa \hbar^{2}}{m_{y}} \partial_{\tau}^{2}+\frac{m_{y} \hbar^{2}}{2} \partial_{y}^{2}+V \mathrm{e}^{y}\right) \Psi(\tau, y)=0,
$$

That again takes the form of Eq. (45) with,

$$
\mathbf{D}=+\frac{\hbar^{2} m_{y}^{2}}{6 \varkappa} \partial_{y}^{2}+\frac{V m_{y}}{3 \varkappa} \mathrm{e}^{y} .
$$

The separation of variables allows us to find the solution using two equations

$\left(\partial_{\tau}^{2}+\omega^{2}\right) f(\tau, \omega)=0$.

and

$$
\left(\frac{m_{y} \hbar^{2}}{2} \partial_{y}^{2}+V \mathrm{e}^{y}\right) \psi(\omega, y)=\frac{3 \varkappa \omega^{2}}{m_{y}} \psi(\omega, y),
$$

The Eq. (71) is very similar to Eq. (60) with $m_{x} V<0$, hence it will give rise to a similar problem, which will be considered in Sect. 2 as well. But in this case the sign of $\omega^{2}$ is different with quintessence, the general solutions include two parts, one is the time-oscillating functions constructed from the discrete spectrum, and the other is decreasing and increasing functions as the superpositions of the modes with continuous spectrum,

$$
\begin{aligned}
\Psi(\tau, y)= & \sum_{( \pm)} \sum_{n=0}^{+\infty} \mathscr{A}_{n}^{( \pm)} \mathrm{e}^{\mp \frac{i}{\hbar} \tau \omega_{n}} \Phi_{n}^{(a)}\left(\frac{2}{\hbar} \sqrt{\frac{2 V}{m_{y}}} \mathrm{e}^{y / 2}\right) \\
& +\int_{-\infty}^{+\infty} \mathrm{d} \tilde{\omega} \mathscr{B}(\tilde{\omega}) \mathrm{e}^{-\frac{1}{\hbar} \tau \tilde{\omega}} \Xi_{\nu}^{(a)}\left(\frac{2}{\hbar} \sqrt{\frac{2 V}{m_{y}}} \mathrm{e}^{y / 2}\right),
\end{aligned}
$$

where,

$2 n+a=\frac{2 \sqrt{6 \varkappa}}{\hbar m_{y}} \omega_{n}, \quad \mu=\frac{2 \sqrt{6 \varkappa}}{\hbar m_{y}} \tilde{\omega}$.

Similarly to the case of quintessence with $m_{x}<0$ and $V>0$ one can exclude the continuous spectrum to preserve both probability conservation and correct classical limit with $\omega^{2}>0$ by setting

$\mathscr{B}(\tilde{\omega})=0$.
The resulting wave packet can be written explicitly as,

$\Psi(\tau, y)=\sum_{( \pm)} \sum_{n=0}^{+\infty} \mathscr{A}_{n}^{( \pm)} \sqrt{2 n+a} \mathrm{e}^{\mp \frac{1}{\hbar} \tau \omega_{n}} \mathrm{~J}_{2 n+a}\left(\frac{2}{\hbar} \sqrt{\frac{2 V}{m_{y}}} \mathrm{e}^{y / 2}\right)$

If the wave packet is only restricted to the positive frequencies the discreteness will be associated with periodicity of $\tau$. The value of $a$ can be fixed by the condition,

$\Psi(\tau, y)=\mathrm{e}^{\mathrm{i} a \pi} \Psi\left(\tau+\frac{2 \pi \sqrt{6 \varkappa}}{m_{y}}, y\right)$

Such periodic condition also guarantees the selfadjointness of the $\partial_{\tau}^{2}$ operator. If both positive and negative frequencies are included the only possibilities are $a=0$ and $a=1$ corresponding to periodic and antiperiodic wavefunctions respectively.

\section{The limit $\lambda \rightarrow 0$}

As a verification of our approach to the minisuperspace trajectory, the limit $\lambda \rightarrow 0$ will be considered, which have been extensively studied as a pedagogic model, see e.g. [5,13,21]. This limit enforces $m_{x}<0$ which will be assumed for the rest of the section.

The action in Eq. (30) in this limit becomes

$S(0)=\int \mathrm{d} t N \mathrm{e}^{3 \alpha}\left(-\frac{3}{\varkappa} \frac{\dot{\alpha}^{2}}{N^{2}}+\sigma \frac{\dot{\phi}^{2}}{2 N^{2}}-V\right)$,

and the integral of motion Eq. (33) tends to

$\omega \rightarrow \sigma \mathrm{e}^{3 \alpha} \frac{\dot{\phi}}{N} \equiv p_{\phi}, \quad \lambda \rightarrow 0$.

For quintessence model with $V<0$, one obtains the classical solution from Eq. (39) by setting $\lambda=0$

$\mathrm{e}^{6 \alpha}=\frac{p_{\phi}^{2}}{2 V} \operatorname{csch}^{2}\left(\sqrt{\frac{3 \varkappa}{2}} \phi+c_{1}\right)$.

The quantum solution can also be calculated in similar way

$$
\begin{aligned}
\Psi(\alpha, \phi)= & \int_{-\infty}^{+\infty} \mathrm{d} p_{\phi} \mathscr{A}\left(p_{\phi}\right) \mathrm{e}^{-\frac{\mathrm{i}}{\hbar} \phi p_{\phi}} \\
& \times\left[c_{1} \mathrm{~F}_{\mathrm{i} \nu}\left(\frac{2}{\hbar} \sqrt{\frac{V}{3 \varkappa}} \mathrm{e}^{3 \alpha}\right)+c_{2} \mathrm{G}_{\mathrm{i} \nu}\left(\frac{2}{\hbar} \sqrt{\frac{V}{3 \varkappa}} \mathrm{e}^{3 \alpha}\right)\right]
\end{aligned}
$$


where the index of the Bessel function becomes

$v=\sqrt{\frac{2}{3 \varkappa}}\left|\frac{p_{\phi}}{\hbar}\right|$.

For the phantom model, one obtains

$\mathrm{e}^{6 \alpha}=\frac{p_{\phi}^{2}}{2 V} \sec ^{2}\left(\sqrt{\frac{3 \varkappa}{2}} \phi+c_{2}\right)$

and

$\Psi(\alpha, \phi)=\sum_{n} \mathscr{A}\left(p_{n}\right) \mathrm{e}^{-\frac{\mathrm{i}}{\hbar} p_{n} \phi} \mathbf{J}_{2 n+a}\left(\frac{2}{\hbar} \sqrt{\frac{V}{3 \varkappa}} \mathrm{e}^{3 \alpha}\right)$.

\section{Semiclassical wave packets and comparisons with classical solutions}

With the explicit form of minisuperspace trajectories at hand, its comparison with the quantum solutions becomes more transparent, since the latter does not depend on any time parameter, but only the minisuperpace coordinates. It is expected that a classical trajectory could be restored from the wave functions at the limit $\hbar \rightarrow 0$, which must be consistent with the results in Sect. (3); furthermore, the cosmological wave packets are expected to go along the classical trajectories in minisuperspace, which can be visualized in plots.

\subsection{WKB limit as $\hbar \rightarrow 0$}

The minisuperspace Wheeler-DeWitt wave functions can be compared with the classical trajectories by taking the WKB limit, i.e. expanding at $\hbar \rightarrow 0$.

For the model with $m_{x} V<0$, it is sufficient to consider the phase contribution of $\mathrm{F}_{\mathrm{i} v}(x)$. The uniform asymptotic expansion of unmodified Bessel function for large index $v$ provides the leading order [40]

$$
\begin{aligned}
\mathrm{F}_{\mathrm{i} v}(v z) & \sim\left(\frac{2}{\pi v}\right)^{1 / 2}\left(1+z^{2}\right)^{-1 / 4} \cos \left(\zeta v-\frac{\pi}{4}\right) \\
\zeta & :=\left(1+z^{2}\right)^{1 / 2}+\ln \left(\frac{z}{1+\left(1+z^{2}\right)^{1 / 2}}\right) .
\end{aligned}
$$

The zeroth-order action reads

$$
\frac{S_{0}}{\hbar}=-\frac{\tau \omega}{\hbar}+v\left[\left(1+z^{2}\right)^{1 / 2}+\ln \left(\frac{z}{1+\left(1+z^{2}\right)^{1 / 2}}\right)\right]-\frac{\pi}{4},
$$

where

$v:=2 \frac{\sqrt{6 x}}{\hbar}\left|\frac{\omega}{m_{x}}\right|, \quad z:=\frac{1}{|\omega|} \sqrt{\frac{-V m_{x}}{3 x}} \mathrm{e}^{x / 2}$.

$\partial S_{0} / \partial \omega=0$ gives

$\mathrm{e}^{6 \alpha+\lambda \phi}=\frac{3 \varkappa \omega^{2}}{-m_{x} V} \operatorname{csch}^{2}\left(\lambda \sqrt{\frac{3}{2 \varkappa}} \alpha+\sqrt{\frac{3 \varkappa}{2}} \phi\right)$,

which is consistent with Eq. (40) up to a choice of $c_{2}$.

For the phantom model, the Bessel function $\mathrm{J}_{n}$ is to be considered, whose leading-order expansion reads

$\mathrm{J}_{v}(v z) \sim\left(\frac{4 \zeta}{1-z^{2}}\right)^{1 / 4} \frac{\mathrm{Ai}\left(v^{2 / 3} \zeta\right)}{v^{1 / 3}}$

for

$\frac{2}{3}(-\zeta)^{3 / 2}=\left(z^{2}-1\right)^{1 / 2}-\arccos \frac{1}{z}, \quad|z|>1$,

$\operatorname{Ai}\left(v^{2 / 3} \zeta\right) \sim \frac{1}{\sqrt{\pi}\left(-v^{2 / 3} \zeta\right)^{1 / 4}} \cos \left(\frac{2}{3} v(-\zeta)^{3 / 2}-\frac{\pi}{4}\right)$.

The zeroth-order of action then reads

$\frac{S_{0}}{\hbar}=\frac{\tau \omega}{\hbar}+v\left[\left(z^{2}-1\right)^{1 / 2}-\arccos \frac{1}{z}\right]-\frac{\pi}{4}$,

where

$v=\frac{2 \sqrt{6 \varkappa}}{\hbar m_{y}}|\omega|, \quad z=\frac{1}{|\omega|} \sqrt{\frac{V m_{y}}{3 \varkappa}} \mathrm{e}^{y / 2}$.

Consequently $\partial S_{0} / \partial \omega=0$ gives us

$\mathrm{e}^{6 \alpha+\lambda \phi}=\frac{3 \varkappa \omega^{2}}{V m_{y}} \sec ^{2}\left(\lambda \sqrt{\frac{3}{2 \varkappa}} \alpha-\sqrt{\frac{3 \varkappa}{2} \phi}\right)$,

which is consistent with Eq. (42).

\subsection{WKB Gaussian wave packet}

The WKB Gaussian wave packet of quintessence models is expected to solve the Wheeler-DeWitt equation in the WKB approximation. For the model with $m_{x} V<0$, one obtains

$\psi^{\mathrm{WKB}}=C(x, \omega) \mathrm{e}^{\frac{\mathrm{i}}{\hbar} S_{0}}$, 
where

$C(x, \omega):=\frac{c}{\sqrt{\left|\partial_{x} S_{0}\right|}}=\frac{c}{\sqrt[4]{f / m_{x}^{2}}}, \quad f:=6 \varkappa \omega^{2}-2 m_{x} V \mathrm{e}^{x}$,

and two zeroth-order actions are

$S_{0}= \pm \frac{2 \sqrt{f}}{m_{x}} \mp \frac{2 \omega \sqrt{6 \varkappa}}{m_{x}} \operatorname{arccoth}\left(\omega \sqrt{\frac{6 \varkappa}{f}}\right)$,

so that a Gaussian wave packet can be written as

$\Psi=\int_{-\infty}^{+\infty} \mathrm{d} \omega \mathscr{A}(\omega, \bar{\omega}) \mathrm{e}^{\frac{\mathrm{i}}{\hbar} \tau \omega} \psi^{\mathrm{WKB}}$,

where $\mathscr{A}(\omega, \bar{\omega})$ is the square root of a Gaussian distribution

$\mathscr{A}(\omega, \bar{\omega})=\frac{1}{(\hbar \Gamma \sqrt{\pi})^{1 / 2}} \exp \left[-\frac{(\omega-\bar{\omega})^{2}}{2 \hbar^{2} \Gamma^{2}}\right]$.

To integrate Eq. (98), one can first expand $S_{0}$ around $\bar{\omega}$,

$S_{0}=\bar{S}_{0}+\left(\partial_{\omega} \bar{S}_{0}\right) \Delta \omega+\frac{1}{2}\left(\partial_{\omega}^{2} \bar{S}_{0}\right) \Delta \omega^{2}+o\left(\Delta \omega^{3}\right)$

then apply the stationary phase approximation, and obtain the general form of wave packet

$\Psi=\sqrt{\frac{\sqrt{\pi}}{\hbar \Gamma}} C(x, \bar{\omega}) \mathrm{e}^{\frac{\mathrm{i}}{\hbar} \alpha \bar{\omega}} \frac{\mathrm{e}^{P^{2} / 4 Q+\frac{\mathrm{i}}{\hbar} \bar{S}_{0}}}{\sqrt{Q}}+\cdots$

The ellipsis denote the same formula but with the another $\bar{S}_{0}$, and

$Q:=\frac{1}{2 \Gamma^{2} \hbar^{2}}-\frac{\mathrm{i}}{2 \hbar} \partial_{\omega}^{2} \bar{S}_{0}, \quad P:=\frac{\mathrm{i}}{\hbar}\left(\tau+\partial_{\omega} \bar{S}_{0}\right)$.

For the phantom model, in the limit $\hbar \rightarrow 0$ the discreteness diappears and we can assume that the spectrum is continuous. Note that this approximation makes the leading order blind to the choice of the self-adjoint extension. The WKB wave packet is $\psi^{\mathrm{WKB}}=C(y, \omega) \mathrm{e}^{\frac{\mathrm{i}}{\hbar} S_{0}}$, with

$S_{0}= \pm \frac{2 \sqrt{f}}{m_{y}} \mp \frac{2 \omega \sqrt{6 x}}{m_{y}} \arctan \left(\frac{1}{\omega} \sqrt{\frac{f}{6 x}}\right)$,

and

$C(y, \omega):=\frac{c}{\sqrt[4]{f / m_{y}^{2}}}, \quad f:=-6 x \omega^{2}+2 m_{y} V \mathrm{e}^{y}$

\subsection{Numerical matching}

The integral with Gaussian distribution in Eq. (98) cannot be implemented analytically. Even though the WKB approximation Sect. (6.2) is effective, its precision is poor in regions where semiclassical approach does not hold, for instance near the classical turning point. Instead, one can turn to numerical approaches.

With the wave functions normalized, one may construct wave packets for the quintessence and phantom models. The corresponding plots are in (2), (3) and (4). The parameters are specified in Planck units $\hbar=\varkappa=1$. The common feature of the plots is that the wave packets coincide with classical trajectories and follow them as closely as possible. The height of the wave 'tube' is negatively correlated to the 'speed' of the classical trajectory with respect to the Klein-Gordon time $\tau$, i.e. the higher the 'speed' is, the lower the amplitude of the wave 'tube' is [53]. It is interesting to note that for all models the naive inner product Eq. (46) happen to approximate the conserved norm Eq. (45) very well so that there's no noticeable difference in plots.

In Figs. 2 and 3, the classical trajectory contains two disjoint branches representing two distinct solutions separated by cosmological singularity. This leads to a quite interesting interference of the two wave tubes. The different choice of $a$ corresponds to slightly different wave packets.

For the phantom model, on the other hand, Poisson's distribution of momentum (see Fig. 4) has been chosen,

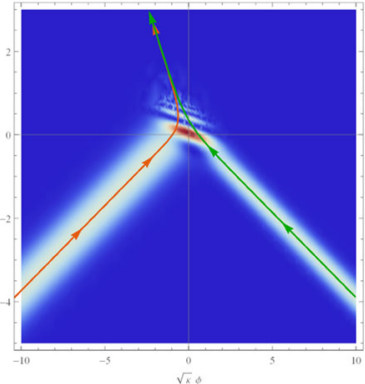

(a) $\mathrm{F}_{\mathrm{i} v}$, Schrödinger inner product

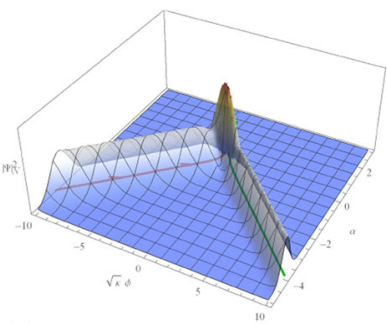

(c) $\mathrm{F}_{\mathrm{i} v}$, Schrödinger inner product

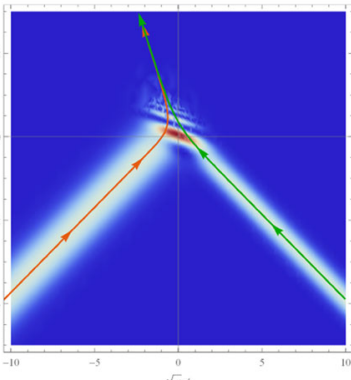

(b) $\mathrm{F}_{\mathrm{i} v}$, Mostafazadeh inner product

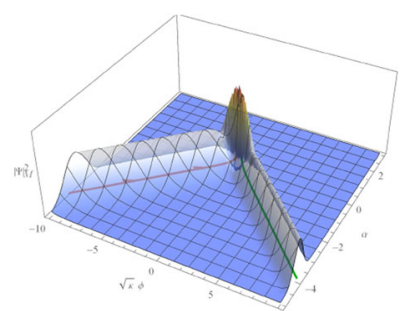

(d) $\mathrm{F}_{\mathrm{i} v}$, Mostafazadeh inner product
Fig. 2 Wave packets and the corresponding classical trajectories for quintessence with $m_{x} V<0$. Parameters are $V=+1, \lambda=4 / 5, \bar{\omega}=$ $-35 / 8$ and $\Gamma=7 / 5$ 


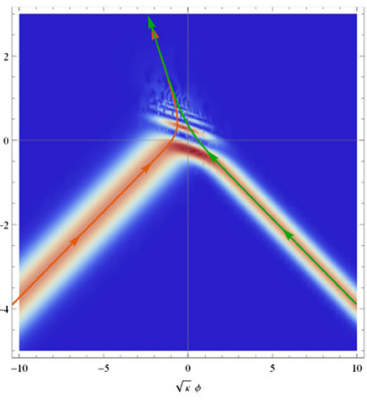

(a) $G_{i v}$, Schrödinger inner product

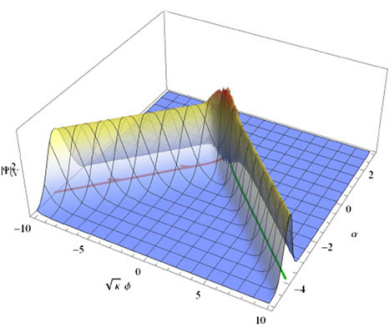

(c) $\mathrm{G}_{\mathrm{iv}}$, Schrödinger inner product

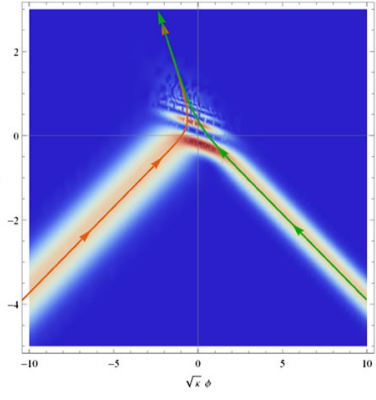

(b) $\mathrm{G}_{\mathrm{iv}}$, Mostafazadeh inner product

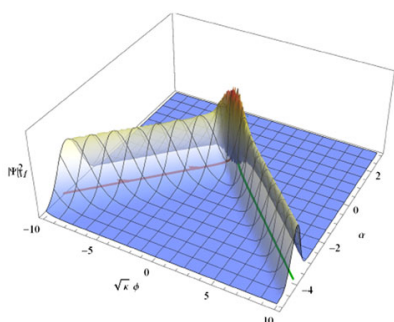

(d) $\mathrm{G}_{\mathrm{i} v}$, Mostafazadeh inner product
Fig. 3 Wave packets and the corresponding classical trajectories for the quintessence with $m_{x} V<0$. Parameters are $V=+1, \lambda=4 / 5$, $\bar{\omega}=-35 / 8$ and $\Gamma=7 / 5$

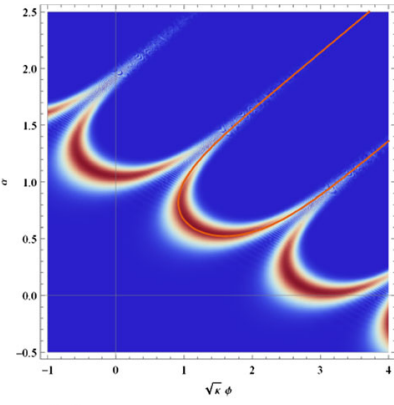

(a) Schrödinger inner product

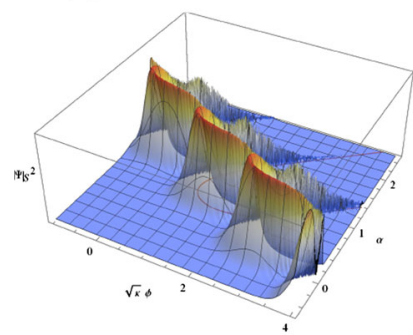

(c) Schrödinger inner product

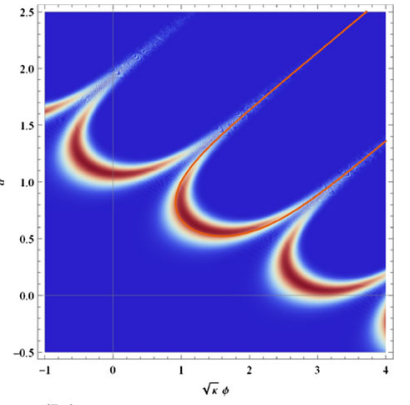

(b) Mostafazadeh inner product

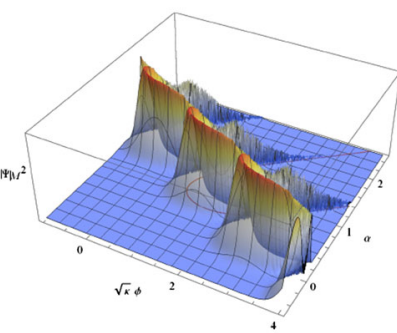

(d) Mostafazadeh inner product
Fig. 4 Poissonian wave packets established by the wave functions with discrete spectrum and the corresponding classical trajectories for the phantom model. Parameters are $V=1, \lambda=2, \bar{\omega}^{2}=10240 / 9$, meanwhile $a=1$ is fixed

$\mathscr{A}_{n}=\frac{\bar{n}^{n / 2} \mathrm{e}^{-\bar{n} / 2}}{\sqrt{n !}}$.

As expected from Eq. (76) the wave packet is periodic in $\tau$ emerging along all the periodic classical solutions separated by Big Rip singularities.

\section{Conclusions}

In this paper, by using the integral of motion to eliminate the lapse function in Friedmann equation, we have solved the cosmological model with Liouville field for homogeneous isotropic metrics. The general classical solutions are obtained and represented in terms of minisuperspace variables only, such that the correspondence between classical and quantum theory can be demonstrated manifestly. The quantum wave packets reproduce the classical limit in a sense that the distributions of traditional Schrödinger's norm and the Mostafazadeh's inner product are maximized near the classical trajectories.

Generally if ordinary matter is added, the model loses integrability and it is not possible to find the analytical solution even in terms of minisuperspace variables. Nevertheless one may consider the integrable model with multiple scalar fields considered in [14]. The homogeneous WheelerDeWitt equation for this model is separated into a system of the Schrödinger equations for one-dimensional particle in the exponential potential. Each of the fields could be then treated independently in the same way as the one-field model considered in this paper. Thus our results are trivially generalized to this integrable model.

The classical models of quintessence with potential unbounded below and the phantom fields give rise to the appearance of a family of non-equivalent quantum models, because the energy density operators are not essentially self-adjoint operator. In order to preserve unitarity and correct classical limit one has to omit half of the spectrum. While this requires that the wave packet at some fixed $\tau$ belongs to much narrower class than $L^{2}(\mathbb{R})$, it is enough to produce wave packets in the vicinity of the classical trajectories.

For the phantom field the resulting spectrum is discrete. It is associated with the fact that at the classical level the universe exists in a finite interval between two singularities and non-singular unitary evolution is accessible through the periodicity of wave function. This periodicity may be regarded as a fundamental condition not only for the homogeneous but also on inhomogeneous modes. On the other hand, if the minisuperspace wave packet contains multiple semiclassical branches they may be associated with coherent superposition of different universes. This Schrödinger-cat-like effect at the cosmic scale might be an artifact of the model in minisuperspace. In the full theory in Wheeler's superspace [54], inhomogeneity is involved, which may serve as an unobservable environment, in contrast with the scale factor [55]. The observable effects are then fully described by the density matrix of the scale factor only, whose off-diagonal elements characterize the superposition of universes with different scale factors. Calculation suggests that those elements are highly-suppressed in the above-mentioned decoherence 
scheme [56,57]; hence the cosmic Schrödinger cat might be fictitious, and the superposition of distinct semiclassical branches might be decohered to vanish. The approach developed in the paper can also be extended to Higher dimensional $[24,25]$ and anisotropic models, such as Bianchi-I cosmology considered in [12].

It is known that the quantum field theory with the phantom fields considered on the classical cosmological background suffers from the vacuum instability problem $[58,59]$. However the self-adjoint issues in the homogeneous modes quantization considered in this paper may influence the applicability of the mean field approach. To address this question one should combine the Wheeler-DeWitt equation on the homogeneous minisuperspace with inhomogeneous perturbations. This could be done for instance starting with the Born-Oppenheimer approximation of the Wheeler-DeWitt equation for the free inhomogeneous perturbations [60-63]. We leave this problem for future work.

As the different self-adjoint extensions lead to different quantum evolution and require the wavefunction to belong to the different restricted functional class they may produce different observable results. The leading order of WKB approximation is insensitive however one may expect that the choice of self-adjoint extension should be important for the NLO corrections to the spectra of perturbations [64-67].

Acknowledgements The funding for this work was provided by the RFBR projects 16-02-00348 and 18-02-00264 (A. A. and O. N.) and the Spanish MINECO under project MDM-2014-0369 of ICCUB (Unidad de Excelencia 'Maria de Maeztu'), Grant FPA2016-76005-C2-1-P, Grant 2014-SGR-104 (Generalitat de Catalunya) (A. A.). Y. -F. Wang is grateful to the Bonn-Cologne Graduate School for Physics and Astronomy (BCGS) for financial support. The authors are grateful to Claus Kiefer for illuminating comments and suggestions as well as to Maxim Kurkov for valuable remarks. Y. -F. Wang is also grateful to Nick Kwidzinski, Dennis Piontek and Tim Schmitz for inspiring discussions.

Open Access This article is distributed under the terms of the Creative Commons Attribution 4.0 International License (http://creativecomm ons.org/licenses/by/4.0/), which permits unrestricted use, distribution, and reproduction in any medium, provided you give appropriate credit to the original author(s) and the source, provide a link to the Creative Commons license, and indicate if changes were made. Funded by SCOAP ${ }^{3}$.

\section{References}

1. A. Linde, Inflationary Cosmology (Springer, Berlin, 2008), pp. 154. https://doi.org/10.1007/978-3-540-74353-8_1

2. E.J. Copeland, M. Sami, S. Tsujikawa, Int. J. Mod. Phys. D 15(11), 1753 (2006). https://doi.org/10.1142/S021827180600942X

3. J.J. Halliwell, Phys. Lett. B 185(3-4), 341 (1987). https://doi.org/ 10.1016/0370-2693(87)91011-2

4. V. Gorini, A.Yu. Kamenshchik, U. Moschella, V. Pasquier, Phys. Rev. D 69(12), 123512 (2004) https://doi.org/10.1103/PhysRevD. 69.123512
5. M.P. Dąbrowski, C. Kiefer, B. Sandhöfer, Phys. Rev. D (2006). https://doi.org/10.1103/PhysRevD.74.044022

6. A.A. Andrianov, F. Cannata, A.Yu. Kamenshchik, J. Cosmol. Astropart. Phys. 2011(10), 004 (2011). https://doi.org/10.1088/ $1475-7516 / 2011 / 10 / 004$

7. A.A. Andrianov, F. Cannata, A.Yu. Kamenshchik, Phys. Rev. D (2012). https://doi.org/10.1103/PhysRevD.86.107303

8. A.Yu. Kamenshchik, E.O. Pozdeeva, A. Tronconi, G. Venturi, S.Y. Vernov, Class. Quant. Grav. 31(10), 105003 (2014). https://doi.org/ 10.1088/0264-9381/31/10/105003

9. A.Yu. Kamenshchik, C.F. Steinwachs, Phys. Rev. D (2015). https:// doi.org/10.1103/PhysRevD.91.084033

10. A.Yu. Kamenshchik, E.O. Pozdeeva, A. Tronconi, G. Venturi, S.Y. Vernov, Class. Quant. Grav. 33(1), 015004 (2015). https://doi.org/ 10.1088/0264-9381/33/1/015004

11. A.Yu. Kamenshchik, E.O. Pozdeeva, S.Y. Vernov, A. Tronconi, G. Venturi, Phys. Rev. D (2016). https://doi.org/10.1103/PhysRevD. 94.063510

12. A.Yu. Kamenshchik, E.O. Pozdeeva, S.Y. Vernov, A. Tronconi, G. Venturi, Phys. Rev. D (2017). https://doi.org/10.1103/PhysRevD. 95.083503

13. A.Yu. Kamenshchik, E.O. Pozdeeva, A. Tronconi, G. Venturi, S.Y. Vernov, Phys. Part. Nucl. Lett. 14(2), 382 (2017). https://doi.org/ 10.1134/S1547477117020169

14. A.A. Andrianov, O.O. Novikov, C. Lan, Theor. Math. Phys. 184(3), 1224 (2015). https://doi.org/10.1007/s11232-015-0328-5

15. A.A. Andrianov, C. Lan, O.O. Novikov, Non-Hermitian Hamiltonians in Quantum Physics. Springer Proceedings in Physics (Springer, Berlin, 2016), pp. 29-44. https://doi.org/10.1007/ 978-3-319-31356-6_3

16. C.M. Chen, P.M. Ho, I.P. Neupane, N. Ohta, J.E. Wang, J. High Energy Phys. 2003(10), 058 (2003). https://doi.org/10.1088/ 1126-6708/2003/10/058

17. N. Ohta, Int. J. Mod. Phys. A 20(01), 1 (2005). https://doi.org/10. 1142/S0217751X05021257

18. A.O. Barvinsky, Phys. Rep. 230(5-6), 237 (1993). https://doi.org/ 10.1016/0370-1573(93)90032-9

19. G. Fulop, D.M. Gitman, I.V. Tyutin, Int. J. Theor. Phys. 38(7), 1941 (1999). https://doi.org/10.1023/a:1026641400067

20. C. Kiefer, Quantum Gravity. International Series of Monographs on Physics, 3rd edn. (Oxford University Press, Oxford, 2012). https://doi.org/10.1093/acprof:oso/9780199585205.001.0001

21. A.O. Barvinsky, A.Yu. Kamenshchik, Phys. Rev. D 89(4), 043526 (2014). https://doi.org/10.1103/PhysRevD.89.043526

22. D.S. Gorbunov, V.A. Rubakov, Introduction to the Theory of the Early Universe: Hot big bang theory, 3rd edn. (World Scientific, Singapore, 2017). https://doi.org/10.1142/10447

23. C. Lan, The integrable cosmological model with scalar field and its extension to PT-symmetric theory. Ph.D. thesis, SPbSU (2016). URL https://search.rsl.ru/ru/record/01006663434

24. A.A. Garcia, S. Carlip, Phys. Lett. B 645(2-3), 101 (2007). https:// doi.org/10.1016/J.PhysLetB.2006.11.064

25. P.S. Letelier, J.P.M. Pitelli, Phys. Rev. D (2010). https://doi.org/10. 1103/PhysRevD.82.104046

26. A.M. Essin, D.J. Griffiths, Am. J. Phys 74(2), 109 (2006). https:// doi.org/10.1119/1.2165248

27. V.S. Araujo, F.A.B. Coutinho, J.F. Perez, Am. J. Phys 72(2), 203 (2004). https://doi.org/10.1119/1.1624111

28. M. Bouhmadi-López, C. Kiefer, B. Sandhöfer, P.V. Moniz, Phys. Rev. D 79(12), 124035 (2009). https://doi.org/10.1103/PhysRevD. 79.124035

29. G. Bonneau, J. Faraut, G. Valent, Am. J. Phys 69(3), 322 (2001). https://doi.org/10.1119/1.1328351

30. D.M. Gitman, I.V. Tyutin, B.L. Voronov, Self-adjoint Extensions in Quantum Mechanics (Birkhäuser, Basel, 2012). https://doi.org/ 10.1007/978-0-8176-4662-2 
31. B.C. Hall, Quantum Theory for Mathematicians, Graduate Texts in Mathematics, vol. 267 (Springer, Berlin, 2013). https://doi.org/ 10.1007/978-1-4614-7116-5

32. C.R. Almeida, A.B. Batista, J.C. Fabris, P.R.L.V. Moniz, Grav. Cosmol. 21(3), 191 (2015). https://doi.org/10.1134/ S0202289315030020

33. S. Gryb, K.P.Y. Thébault (2018). arXiv:1801.05789 [gr-qc]

34. A. Mostafazadeh, Class. Quant. Grav. 20(1), 155 (2002). https:// doi.org/10.1088/0264-9381/20/1/312

35. A. Mostafazadeh, F. Zamani, Ann. Phys. 321(9), 2183 (2006). https://doi.org/10.1016/j.aop.2006.02.007

36. A. Mostafazadeh, Int. J. Geom. Methods Mod. Phys. 07(07), 1191 (2010). https://doi.org/10.1142/s0219887810004816

37. C. Kiefer, Nucl. Phys. B 341(1), 273 (1990). https://doi.org/10. 1016/0550-3213(90)90271-e

38. C. Kiefer, Phys. Rev. D 38(6), 1761 (1988). https://doi.org/10. 1103/PhysRevD.38.1761

39. C. Kiefer, H.D. Zeh, Phys. Rev. D 51(8), 4145 (1995). https://doi. org/10.1103/PhysRevD.51.4145

40. T.M. Dunster, SIAM J. Math. Anal. 21(4), 995 (1990). https://doi. org/10.1137/0521055

41. R. Szmytkowski, S. Bielski, J. Math. Anal. Appl. 365(1), 195 (2010). https://doi.org/10.1016/j.jmaa.2009.10.035

42. G.N. Watson, A Treatise on the Theory of Bessel Functions (Cambridge University Press, Cambridge, 1922)

43. F.W.J. Olver, D.W. Lozier, R.F. Boisvert, C.W. Clark, NIST Handbook of Mathematical Functions Hardback and CD-ROM (Cambridge University Press, Cambridge, 2010)

44. R.R. Caldwell, R. Dave, P.J. Steinhardt, Phys. Rev. Lett. 80(8), 1582 (1998). https://doi.org/10.1103/PhysRevLett.80.1582

45. R.R. Caldwell, Phys. Lett. B 545(1-2), 23 (2002). https://doi.org/ 10.1016/s0370-2693(02)02589-3

46. P.A.M. Dirac, Lectures on Quantum Mechanics, Belfer Graduate School of Science Monographs Series, vol. 2 (Yeshiva University, New York, 1964)

47. D.M. Gitman, I.V. Tyutin, Quantization of Fields with Constraints. Springer Series in Nuclear and Particle Physics (Springer, Berlin, 1990). https://doi.org/10.1007/978-3-642-83938-2

48. H.J. Rothe, K.D. Rothe, Classical and Quantum Dynamics of Constrained Hamiltonian Systems. World Scientific Lecture Notes in Physics (World Scientific, Singapore, 2010). https://doi.org/10. $1142 / 7689$

49. M. Henneaux, C. Teitelboim, Quantization of Gauge Systems (Princeton University Press, Princeton, 1994). http://press. princeton.edu/titles/5156.html. Accessed 21 Sept 2018
50. L.V. Prokhorov, S.V. Shabanov, Hamiltonian Mechanics of Gauge Systems (Cambridge University Press, Cambridge, 2009). https:// doi.org/10.1017/cbo9780511976209

51. D.S. Salopek, J.R. Bond, Phys. Rev. D 42(12), 3936 (1990). https:// doi.org/10.1103/PhysRevD.42.3936

52. B.S. DeWitt, Phys. Rev. 160(5), 1113 (1967). https://doi.org/10. 1103/PhysRev.160.1113

53. S.W. Hawking, D.N. Page, Nucl. Phys. B 264, 185 (1986). https:// doi.org/10.1016/0550-3213(86)90478-5

54. J.A. Wheeler, in Battelle Rencontres, ed. by C.A.P. DeWittMorette, J.A. Wheeler (Benjamin, New York, 1968), pp. 242-307

55. C. Kiefer, in Towards Quantum Gravity, vol. 541, Lecture Notes in Physics, ed. by J. Kowalski-Glikman (Springer, Berlin, 2000), pp. 158-187. https://doi.org/10.1007/3-540-46634-7_7

56. C. Kiefer, Class. Quant. Grav. 4(5), 1369 (1987). https://doi.org/ 10.1088/0264-9381/4/5/031

57. C. Kiefer, Phys. Rev. D 46(4), 1658 (1992). https://doi.org/10. 1103/PhysRevD.46.1658

58. I.Ya. Aref'eva, I.V. Volovich, Theor. Math. Phys. 155(1), 503 (2008). https://doi.org/10.1007/s11232-008-0041-8

59. R. Kallosh, J.U. Kang, A. Linde, V. Mukhanov, J. Cosmol Astropart. Phys. 2008(04), 018 (2008). https://doi.org/10.1088/ $1475-7516 / 2008 / 04 / 018$

60. A.Yu. Kamenshchik, A. Tronconi, G. Venturi, Phys. Lett. B726, 518 (2013). https://doi.org/10.1016/j.physletb.2013.08.067

61. A.Y. Kamenshchik, A. Tronconi, G. Venturi, Phys. Lett. B734, 72 (2014). https://doi.org/10.1016/j.physletb.2014.05.028

62. A.Yu. Kamenshchik, A. Tronconi, G. Venturi, Class. Quant. Grav. 35(1), 015012 (2018). https://doi.org/10.1088/1361-6382/aa8fb3

63. O.O. Novikov, Phys. Part. Nucl. Lett. 15(4), 353 (2018). https:// doi.org/10.1134/S1547477118040179

64. C. Kiefer, M. Krämer, Phys. Rev. Lett. 108(2), 021301 (2012). https://doi.org/10.1103/PhysRevLett.108.021301

65. D. Bini, G. Esposito, C. Kiefer, M. Krämer, F. Pessina, Phys Rev. 87(10), 104008 (2013). https://doi.org/10.1103/PhysRevD. 87.104008

66. D. Brizuela, C. Kiefer, M. Krämer, Phys. Rev. D93(10), 104035 (2016). https://doi.org/10.1103/PhysRevD.93.104035

67. D. Brizuela, C. Kiefer, M. Krämer, Phys. Rev. D94(12), 123527 (2016). https://doi.org/10.1103/PhysRevD.94.123527 